\title{
EL BUEN RENDIMIENTO ESCOLAR EN LOS ESTUDIANTES QUE INGRESAN A LA UNIVERSIDAD A TRAVÉS DEL PROGRAMA PROPEDÉUTICO: UN ANÁLISIS DESDE LA MOTIVACIÓN Y EL DISCURSO DE LA UCSH ${ }^{1}$
}

\section{Carola Román Pérez}

\section{RESUMEN}

El presente trabajo tiene como propósito evidenciar las características motivacionales y personales de los estudiantes que cursan bachillerato y cuyo ingreso ha sido a través del programa propedéutico implementado en la Universidad Católica Silva Henríquez (UCSH). Se han aplicado las escalas de automotivación (Martínez, 2011a) y la escala de motivación de logro (Manassero y Vázquez, 1997; 1998). Para el análisis de discurso se ha utilizado la técnica del «análisis estructural». Los resultados muestran que los jóvenes que ingresan a la universidad a través del programa propedéutico presentan igual grado de motivación que los jóvenes que ingresan por la vía tradicional. Asimismo, se aprecia que los jóvenes declaran que detrás de su buen rendimiento existe esfuerzo, planificación y metas claras.

Palabras clave: Desigualdades educativas, motivación de logro, acceso a la educación superior, inclusión en educación superior.

\section{GOOD PERFORMANCE OF STUDENTS ENTERING UNIVERSITY THROUGH THE PROPAEDEUTIC PROGRAM: ANALYSIS OF MOTIVATION AND DISCOURSE IN UCSH}

\section{ABSTRACT}

This paper is aimed to highlight the motivational and personal characteristics of students enrolled in bachelor's degrees and entering through propaedeutic program implemented in Universidad Católica Silva Henríquez (UCSH). It has applied motivation scales (Martínez, 2011a) and achievement motivation scale (Manassero and Vazquez, 1996). For discourse analysis, it has used the «structural analysis» technique. Results show that young people who enter through propaedeutic program have the same level of motivation than those who enter through the traditional way. It also shows that young people declare that behind good performance there are effort, planning and clear goals.

Keywords: Educational inequalities, achievement motivation, access to higher education, including higher education

1 Este estudio forma parte de la investigación «Motivación y expectativas para el aprendizaje efectivo en estudiantes de enseñanza secundaria y universitaria, de contextos socioeconómicos vulnerables que participan en Programa Propedéutico y Bachillerato en Educación Superior UCSH». Financiada por Conicyt a través del proyecto de Inserción de Capital Humano en la Academia n 79100022.

2 Doctora en Ciencias de la Educación. Investigadora de la Universidad Católica Silva Henríquez, UCSH, Programa Propedéutico y Bachillerato, Santiago, Chile. Contacto: groman@ucsh.cl 


\section{EL BUEN RENDIMIENTO ESCOLAR EN \\ LOS ESTUDIANTES QUE INGRESAN A LA \\ UNIVERSIDAD A TRAVÉS DEL PROGRAMA \\ PROPEDÉUTICO: UN ANÁLISIS DESDE LA \\ MOTIVACIÓN Y EL DISCURSO DE LA UCSH}

\section{Problema}

En el contexto de la reforma educativa chilena se han verificado una serie de cambios políticos materializados en leyes, decretos, reglamentos, programas, proyectos y orientaciones que han intentado dar respuesta al compromiso que el país y el Ministerio de Educación (Mineduc,de aquí en adelante) han suscrito en pactos con diversos organismos internacionales para la superación de la desigualdad, la disminución del analfabetismo, la exclusión y la pobreza. Se trata de mejorar la calidad de educación como pilar fundamental para el desarrollo del país.

Sin embargo, es necesario seguir revisando y trabajando en estos planteamientos, especialmente en lo referido a la equidad y a la inclusión. Por ejemplo, en el sistema terciario, el acceso a la educación superior, y las desigualdades que en este sentido se producen, representa un punto de discusión y análisis ampliamente documentado, ya que se considera que la Prueba de Selección Universitaria, PSU, ha marcado las posibilidades de ingreso a la universidad de los jóvenes a partir de su origen socioeconómico y del tipo de establecimiento de procedencia (Kremerman, 2007; OCDE, 2009). Para dar cuenta de esta situación, basta con señalar que mientras tres de cada cuatro alumnos pertenecientes al 20\% más rico de los hogares chilenos accede a la educación superior, solo uno de cada siete alumnos pertenecientes al quintil más pobre se encuentra en igual situación.

Asimismo, los datos del Departamento de Evaluación, Medición y Registro Educacional, DEMRE (2011), evidencian que el 43,2\% de los alumnos que rinde la PSU obtiene menos de 450 puntos, es decir, no obtienen el puntaje mínimo que se requiere para postular a las 
Universidades del Consejo de Rectores. El 62\% de estos estudiantes proviene de colegios municipales. Estos bajos resultados no solo afectan la selección para el ingreso a la universidad, sino que además representan un factor determinante para la distribución de créditos y becas, pues para optar a ellos se exige un mínimo de 475 puntos en la PSU.

Para comprender esta situación es importante revisar en qué consiste y cuáles son los fines de la PSU. Para responder al primer punto se debe tener en cuenta que para acceder a las universidades chilenas los estudiantes son elegidos a partir de los resultados en dicha prueba de selección, la cual consiste en la aplicación de una batería de test que miden habilidades cognitivas en las materias de Lenguaje y Comunicación, Matemática, Historia y Ciencias Sociales y Ciencias. El puntaje que los estudiantes obtienen se pondera con el promedio de notas de Educación Media (NEM). Al NEM se le asigna una ponderación mínima de 20\%, según lo establecido por el Consejo de Rectores de las Universidades Chilenas, CRUCH. El porcentaje restante se distribuye de acuerdo con el valor que asigne cada universidad y carrera a los resultados de la PSU (Donoso y Schiefelbein, 2007).

Continuando con Donoso y Schiefelbein, la instalación de un sistema de selección de estudiantes, por más de siete décadas se sustenta en la premisa de que no todos los egresados de la enseñanza secundaria cuentan con las habilidades, condiciones, capacidades, aptitudes o competencias para concluir estudios universitarios, por lo tanto, la prueba de selección y el NEM se estarían considerando como los únicos y mejores predictores de éxito académico, entendiendo dicho éxito como la retención y el egreso de la universidad.

Sin embargo, aún no se cuenta con evidencia suficiente para asegurar que tanto la PSU como el NEM sean los únicos y mejores predictores de retención en la educación superior. En efecto, recientes investigaciones realizadas en Chile concluyen que aquellos estudiantes que obtienen los mejores promedios de notas en sus respectivos establecimientos ${ }^{3}$ logran mejores desempeños al ingresar a

3 Controlando por PSU y el NEM. 
la educación superior. En consecuencia, lo que proponen los autores es incorporar el «sistema de ranking» como una vía de ingreso a la universidad (Gallegos y Meneses, 2007; Contreras, Gallegos y Meneses, 2009; Meneses y Toro, 2012).

Según los autores antes mencionados, el ranking consiste en ordenar a los alumnos de acuerdo con su rendimiento en la enseñanza media y con el de sus compañeros de egreso del mismo establecimiento escolar. Esta medida de habilidad relativa compara al alumno respecto de sus pares (que se desenvuelven en un mismo contexto y entorno), por lo que podría ser indicativa respecto de las proyecciones académicas del estudiante. Sin embargo, es posible que aunque el ranking efectivamente entregara dicha información, esta ya haya sido identificada por los elementos de la batería de selección universitaria.

En Chile, las aplicaciones del sistema de ranking comenzaron a utilizarse en los programas propedéuticos a partir del año 2007. Esta iniciativa surge en la Universidad de Santiago de Chile (USACH) y luego se ha hecho extensiva a otros planteles educativos. Se trata de programas que tienen como propósito la inclusión de estudiantes provenientes de contextos vulnerados. Esta modalidad de selección se sustenta en el principio de que los talentos y capacidades intelectuales están equitativamente distribuidos entre los distintos grupos sociales, en consecuencia, en cada barrio y en cada establecimiento de enseñanza media, deben existir estudiantes con capacidades y talentos para cursar con éxito estudios universitarios (Gil y Bachs, 2008).

Además, a partir del año 2013 el sistema de ranking, también ha sido incorporado como criterio de selección a las universidades, junto con la PSU y con el NEM. Aun así, esta incorporación se encuentra en pleno debate debido a que, para algunas autoridades del Mineduc, no existe evidencia suficiente de que este sistema apunte a la equidad. En cambio, el CRUCH, apoyándose en estudios de la UNESCO, ha expresado que 4.000 jóvenes lograron entrar a la universidad gracias a este sistema.

Más allá de estos debates, los programas propedéuticos se han difundido en Chile y su premisa se sustenta en la inclusión de 
estudiantes talentosos académicamente provenientes de contextos vulnerables, en el entendido de que estos también tienen derecho a acceder a la educación superior. Si bien, dichos estudiantes se han destacado por su alto rendimiento escolar, poco se sabe respecto de sus características personales y sus trayectorias educativas.

Por ello, el presente trabajo da cuenta de las características motivacionales que presentan estos jóvenes, pues se infiere que existen habilidades que no son captadas por la PSU y que serían causales de este alto rendimiento: habilidades y características no cognitivas que también son necesarias para acceder y egresar de la educación superior (Killonen, 2012). Algunas de las interrogantes que surgen son: ¿cómo se presentan y representan estos jóvenes? ¿Cómo es su motivación hacia el aprendizaje en relación con sus pares cuyo ingreso es a través de la PSU? ¿Por qué los programas propedéuticos y el sistema de ranking son acciones que favorecen la inclusión y la equidad? Son algunas de las preguntas que se responden en la presente investigación realizada a partir de las experiencias de estudiantes pertenecientes a la Universidad Católica Silva Henríquez (UCSH) que han cursado estudios en el programa propedéutico.

\section{Discusión bibliográfica y perspectivas teóricas}

\subsection{Inclusión y acceso a la educación superior en América Latina}

Se aprecia en América Latina un sistema de educación superior con un alto grado de desigualdad. En efecto, en la investigación realizada por Sverdlick, Ferrari y Jaimovich (2005) se sostiene que se trata de sistemas excluyentes desde el punto de vista social por sus propias características internas, las que a su vez se asientan sobre otra exclusión. Es decir, se alude a un tipo de educación superior que selecciona a un conjunto de personas dentro de un universo que ya pasó por fuertes procesos de selección social y educativa en periodos escolares previos.

En consecuencia, el ingreso y permanencia en las universidades perpetúa la selección y estratificación social, puesto que se encubren 
las diferencias que existen entre los representantes de los diversos estratos sociales. Así, los estudiantes del nivel educativo mencionado se relegan o son relegados por la institución educativa, para dar paso a los «herederos» de las clases sociales medias y altas (Bourdieu y Passeron, 1964).

Por otra parte, a pesar de estas críticas también se sostiene que América Latina se encuentra en la «tercera reforma de la educación superior» (Rama, 2006). En efecto, el autor afirma que la «primera reforma» se produjo a comienzos del siglo XX y estuvo marcada por la Reforma de Córdoba en 1918. Dicho proceso contribuyó a la expansión de la cobertura de las universidades públicas, superando los modelos de élite y contribuyendo a la movilidad social. La «segunda reforma» se verifica producto de las dificultades de los Estados para financiar los gastos de la educación superior, lo cual dio pie a la creación de instituciones privadas y a su masificación. Ello tuvo como consecuencia el establecimiento de un modelo dual, con una fuerte diferenciación entre una educación pública (cada vez más elitista socialmente) con restricciones de acceso sobre la base de cupos y exámenes y, por otro, un sector privado capaz de pagar, con restricciones de acceso debido a los costos. Esta situación no afectó la demanda, pero sí la calidad de la educación, haciendo fracasar aquellas creencias de los años ochenta en las cuales se pensaba que el libre mercado podía promover altos niveles de calidad, sin control.

Lo anterior derivó en una fuerte necesidad de políticas públicas destinadas a regular el funcionamiento de las instituciones universitarias. Adicionalmente, se produce el desarrollo de las sociedades a escala global, en el marco de la sociedad del conocimiento y el desarrollo de las tecnologías de la información y de la comunicación y la creciente internacionalización de la educación superior. Situaciones como las descritas fueron la base para el inicio de la «tercera reforma», lo que ha implicado la existencia de motores que impulsan en la actualidad la masificación, la feminización, la privatización, la regionalización, la diferenciación y segmentación de la educación en América Latina, además, también se visualizan los nuevos roles de los estudiantes, por ejemplo, el estudiante como cliente, estudiantes de corto plazo, estudiantes profesionales, 
estudiantes trabajadores, estudiantes a distancia, estudiantes con discapacidades, entre otros. A todo ello se agregan los niveles de competencia en los mercados laborales y la disposición de las familias a sacrificar tiempo y renta para capacitarse.

Este escenario configura nuevos desafíos, entre ellos, la inclusión en educación superior. Según Chiroleu (2009), el desarrollo de políticas que vayan en este sentido supone el desafío de superar la contradicción de su aplicación a un ámbito tradicionalmente refractario a las tendencias igualitaristas. Para la autora, la primacía como la excelencia y el mérito (ambos conceptos propios de la educación superior) se asocian con lógicas excluyentes, ya que focalizan en el individuo, tal como se expresa a continuación:

Se considera que las personas son premiadas por sus acciones y sus logros, los cuales se ligan al esfuerzo, la perseverancia y el trabajo constante. Desde esta perspectiva se sostiene que independientemente su origen y situación inicial, cualquier persona dispuesta a someterse a ese esfuerzo alcanzaría las mismas metas. Para otros, en cambio, este planteo encierra una falsa igualdad de oportunidades, pues el mérito no es un concepto universal y se halla mediado por factores adscriptos por lo que se merece (Chiroleu 2009: 6).

Desde esta perspectiva las políticas públicas inclusivas en educación superior adquieren diferentes significados. Sin embargo, existe cierta representación común en la cual inclusión se entiende como igualdad de oportunidades en el acceso, con algunos programas dirigidos a minorías étnicas y personas con discapacidad y con programas de créditos y becas en los cuales la demanda supera a la oferta. Particularmente, en Argentina se privilegia un alto grado de cobertura, producto de la articulación entre el ingreso y la gratuidad. En Brasil, en tanto, se incluyen sectores tradicionalmente excluidos, sin embargo, este proceso se realiza principalmente en instituciones privadas. En Venezuela se ha intentado implementar un sistema que incluya a los diversos grupos sociales, no obstante, este funciona de manera paralela al tradicional y aún no existen garantías de resultados positivos. 
Podría decirse que en Chile la inclusión también es comprendida como acceso. Sin embargo, el sistema de selección, tal como se ha señalado en apartados anteriores, sigue siendo excluyente.

Esta situación encuentra su principal origen en las desigualdades económicas y sociales. Desigualdades que, por cierto, persisten y se acentúan (Donoso y Schiefelbein, 2009; Redondo, 2009; OCDE y BID, 2009). Los intentos por superar esta situación han venido de parte de todos los actores educativos, aunque tal vez el caso más emblemático se encuentre en la movilización de los estudiantes secundarios realizada durante el año 2006. Este movimiento, denominado «Revolución de los pingüinos» fue la forma a través de la cual los estudiantes evidenciaron plena conciencia frente a los pálidos cambios que realizaron las nuevas autoridades políticas desde 1990 en materias de justicia social y derechos perdidos en el país, como consecuencia de las políticas implementadas en los años ochenta, especialmente, en materia educativa (Abarca, Assáel, Brzovic, Caldichoury, Cornejo, González, Redondo, Sánchez, Sobarzo, 2009).

Este grupo de jóvenes supo articular estratégicamente sus necesidades urgentes (infraestructura, pase escolar, becas de alimentación y PSU, contenidos curriculares, etc.) con demandas de sentido y estructurales (fin a la LOCE, no al lucro con fondos públicos, sistema público con presencia del Estado, no a la selección y segmentación escolar, calidad educativa para todos, etc.) (Redondo 2009: 26).

Las problemáticas fueron nuevamente expuestas por movimientos estudiantiles en el año 2011, cuando los jóvenes protagonizaron acciones en pro de discutir las dificultades educativas y retomar las demandas realizadas en el año 2006. Este segundo movimiento mantuvo un petitorio que, según Urra (2012), se articuló en torno a los siguientes ejes:

a) Financiamiento: traducido en un aumento de aportes basales de libre disposición, no asociados a convenios de desempeño; creación de una red nacional de educación técnica estatal; un fondo de revitalización para universidades de libre disposición. 
b) Democratización y regularización del sistema de educación superior: aquí se destacan las intenciones de garantizar el derecho a la participación triestamental en los cupos colegiados, en las elecciones de autoridades unipersonales y en la toma de decisiones de todas las instituciones de educación superior; aseguramiento de la libertad de expresión, cátedra y de asociación a estudiantes, docentes y trabajadores; prohibición efectiva de lucro mediante la creación de la superintendencia de educación en todo el sistema de educación superior.

c) Acceso con equidad, calidad, integración y heterogeneidad social en la matrícula: se destaca aquí el rechazo a la PSU por su carácter regresivo, elitista, que solo detecta el nivel socioeconómico; y la creación de mecanismos complementarios de acceso que consideren la nivelación, mantención y titulación, para que exista igualdad de oportunidades para todos.

En consecuencia, se está en presencia de un escenario que consolida tanto la desigualdad como la exclusión económica, social y educativa en el ingreso a las universidades chilenas. Este problema tiene su origen, según los autores antes mencionados, en dos causas: por una parte, los altos costos de las carreras universitarias, por lo cual los grupos que presentan carencias económicas no pueden solventar la educación superior; y, en segundo término, la PSU como única vía de acceso a la educación superior y cuyo puntaje es considerado, además, para la distribución de créditos y becas por parte del Estado.

Por su parte, Manzi (2012) en una entrevista publicada en el diario "El Mercurio" sostiene que las inequidades no provienen de la PSU como sistema de selección para el ingreso a la educación superior, ya que esta prueba evidencia lo mismo que todas las mediciones que se aplican en Chile: «(...) el rendimiento está estrechamente correlacionado con el nivel socioeconómico», por lo tanto, el problema no se encuentra en los test, sino en las profundas desigualdades de la sociedad nacional y en los sistemas de selección para ingresar a las universidades. El autor afirma que no se puede exigir equidad a las pruebas, ya que la equidad debe estar en los sistemas de selección. Su propuesta incluye indicadores que no estén correlacionados con el origen social de los estudiantes, por ejemplo, el ranking de notas en 
el colegio de origen de los postulantes. Es por esta razón que Manzi propone los programas propedéuticos como una vía válida de acceso a la universidad.

\subsection{Los programas propedéuticos en Chile $^{4}$}

Los programas propedéuticos tienen como propósito incluir en la educación superior a aquellos jóvenes que obtienen altas calificaciones en la enseñanza secundaria, sin considerar el puntaje que obtengan en la PSU. Algunos de sus principios orientadores son los siguientes:

- Los talentos están igualmente distribuidos entre ricos y pobres.

- La educación es una herramienta de fuerte movilidad social, además de proveer mayores oportunidades de desarrollo personal y de convivencia democrática.

- Las notas de Educación Media son un mejor predictor de rendimiento académico que las pruebas nacionales de selección como la PSU.

- La PSU replica las condiciones de inequidad y exclusión social.

- Hablar de acceso a la educación superior es incompleto, pues la verdadera justicia social se produce al egreso. Se deben aumentar los programas de apoyo que eviten la deserción de los estudiantes, así como también se deben respetar sus particularidades socioculturales.

- Los programas de acción afirmativa son necesarios para trabajar situaciones de exclusión social en condiciones de vulnerabilidad.

- Sentir y palpar las oportunidades reales de desarrollo personal actúa como una gran potencia motivadora.

- Las personas que provienen de medios vulnerables requieren de un periodo de tiempo para nivelar sus resultados académicos. Estas personas presentan indicadores de retención mayor a otros grupos.

Por otra parte, los programas propedéuticos tienen su origen fundacional en el año 2007, en la Universidad de Santiago de Chile (USACH). En dicha instancia, el trabajo tuvo como principal foco de

4 Para mayor información acerca de los programas propedéuticos se sugiere revisar el sitio http://www.propedeutico.cl/ 
atención a los estudiantes de $4^{\circ}$ año de enseñanza media pertenecientes a cuatro programas de liceos prioritarios del Ministerio de Educación de Chile, en asociación con OREALC-UNESCO, Fundación Equitas y Fundación Ford.

Posteriormente, en el año 2009 se adhieren a esta iniciativa de los programas propedéuticos la Universidad Católica Silva Henríquez (UCSH)y la Universidad Alberto Hurtado (UAH). A partir de 2010, esta vía de acceso a la educación superior es implementada en la Universidad Tecnológica Metropolitana (UTEM), en la Universidad Metropolitana de Ciencias de la Educación (UMCE) y en la Universidad Católica de Temuco (UCT). Estas instituciones fueron las primeras en conformar la «Red de Universidades Propedéutico UNESCO».

Entre el año 2011 y 2012 se han incorporado a esta red la Universidad de Antofagasta (UA), Universidad Católica del Norte (UCN), Universidad de Los Lagos (ULA), Universidad Austral de Chile (UACH), la Universidad de Viña del Mar (UVM) y la Universidad Católica de la Santísima Concepción (UCSC).

También es importante considerar que, aun cuando no formen parte de esta red de programas propedéuticos, existen otros planteles de educación superior que también han incorporado programas para favorecer el acceso a sus instituciones. Tal es el caso de la Universidad de Chile (UCHILE), Universidad Diego Portales (UDP) y el Instituto Profesional INACAP.

Finalmente, durante el año 2013 la Universidad Técnica Federico Santa María (UTFS) implementará un programa de acceso a la educación superior dirigido a estudiantes de contextos vulnerables y con alto rendimiento académico.

Si bien es cierto las modalidades y características de los programas propedéuticos difieren de una institución a otra, en general, comparten las siguientes acciones:

- Invitación y selección de los estudiantes que se encuentran cursando $4^{\circ}$ año de enseñanza media y que pertenecen al 5\% o $10 \%$ superior en promedio de notas (ranking) en sus respectivos establecimientos. 
- Clases de nivelación durante el segundo semestre los sábados con participación en cursos de lenguaje y comunicación, matemáticas y gestión personal.

- Los estudiantes que cursan el programa propedéutico tienen la posibilidad de ingresar al programa de bachillerato y luego de un año, ingresar a una carrera universitaria.

Particularmente en lo que se refiere a la experiencia descrita en este documento, el programa propedéutico de la UCSH funciona desde 2009. Es una instancia gratuita de formación y selección de estudiantes, cuyo propósito es que accedan a la educación superior, sin requerimiento de un puntaje mínimo en la PSU y en la cual los participantes deben aprobar un plan de estudios que pone en juego sus destrezas y habilidades.

El objetivo central de este programa es generar nuevas oportunidades para los estudiantes talentosos y que ello contribuya a disminuir las brechas sociales, pues se sustenta en el convencimiento de que los talentos se encuentran igualmente distribuidos entre los jóvenes, independientemente del sector socioeconómico de origen y del colegio en el cual estudian.

Son invitados a participar en este programa los estudiantes de $4^{\circ}$ año medio de la «Red de Colegios Propedéuticos UCSH» que, al finalizar el primer semestre, hayan obtenido un promedio de notas que los ubique en el $5 \%$ superior de su establecimiento educacional. Los egresados del propedéutico UCSH pueden ingresar al Bachillerato en Ciencias y Humanidades, cumpliendo con los siguientes requisitos:

- Aprobar los tres módulos del plan de estudios con calificación igual o superior a 4.0 y un $100 \%$ de asistencia.

- Haber rendido la PSU (no se exige puntaje mínimo).

- Haber obtenido la Beca de Excelencia Académica del Mineduc.

En el año 2013 el grupo de estudiantes que ingresó al programa de Bachillerato en Ciencias y Humanidades, modalidad vespertina, obtuvo un promedio PSU de 499 puntos. Por su parte, los estudiantes que ingresaron a la modalidad diurna obtuvieron 
506 puntos. El promedio PSU de ambos grupos es de 502 puntos. El puntaje promedio PSU en la UCSH para el conjunto de estudiantes que ingresan por la vía tradicional es de 528 puntos, una diferencia de apenas 26 puntos superior. Esta situación es relevante, pues las diferencias entre los estudiantes que ingresan a la universidad vía propedéutico, o bien, por ingreso tradicional son mínimas, lo cual podría dar cuenta de cierta homogeneidad en los perfiles de ambos grupos, por lo tanto, este punto debe ser considerado a la hora de hacer conclusiones que vayan en esta dirección.

Respecto del desempeño y aporte de estos programas en la retención de los estudiantes, las investigaciones que se realizan son recientes y la mayoría se encuentra en desarrollo; sin embargo, es importante considerar algunos de sus resultados preliminares. Por ejemplo, Scheele y Treviño (2012) plantean que los estudiantes que ingresan a la universidad a través del programa propedéutico mantienen un rendimiento similar al de sus pares que ingresan por la vía tradicional y, además, cumplen con las exigencias de la carrera. Sin embargo, la retención en el caso de los jóvenes que ingresan vía propedéutico es menor que sus pares de ingreso tradicional.

Gil y Del Canto (2012) sostienen que los datos que refieren al rendimiento académico de los estudiantes matriculados en dos de las mejores universidades del país (PUC y USACH) y que se encuentran en el 10\% superior del ranking, muestran un desempeño significantemente mejor y tienden a graduarse de manera más oportuna que sus pares del mismo nivel socioeconómico que no se encuentran en este $10 \%$. Si se compara a los estudiantes que ingresan a través del propedéutico con sus pares que ingresan vía PSU se obtiene que los primeros tienen un rendimiento más bajo durante el primer año de estudios, sin embargo, estas dificultades son superadas en el segundo año. Pese a todo, los resultados preliminares también arrojan que los jóvenes ingresados por medio del programa propedéutico se gradúan con un año de retraso.

Leyton, Fuenzalida y Vásquez (2012) sostienen que existen diferencias importantes entre las universidades que cuentan con programas propedéuticos y las que no lo presentan. Concluyen que 
estos programas han tenido un efecto positivo en las experiencias de los estudiantes provenientes de contextos vulnerables, situación que favorece su permanencia en las instituciones de educación superior. En palabras de sus autores:

En efecto, la política de inclusión a través del programa propedéutico interviene como un dispositivo de socialización e incorporación de herramientas de conocimiento teórico-prácticas necesarias en la universidad, con lo cual se suaviza el encuentro entre las disposiciones individuales (asociadas a bajos capitales culturales) y el espacio institucional cercano al manejo de la alta cultura reconocidos por las élites (Leyton, Fuenzalida y Vásquez, 2012: 84).

Tal como se ha señalado en apartados anteriores, estos programas se sustentan especialmente en el sistema de ranking, razón por la cual existen posiciones que relativizan la real igualdad de oportunidades que estos declaran, ya que, finalmente, enmascaran las barreras estructurales que se presentan el sistema social y educativo (Acuña y Arévalo et al. 2009).

Además, Castillo y Cabezas (2010) sostienen que en Chile es necesario modificar la idea de equidad instalada en la educación superior, ya que ella se enfoca más bien en el acceso a dicha enseñanza y no en la calidad de los procesos y resultados que genera: «El éxito o fracaso académico no dependen solo de lo que los estudiantes hacen o dejan de hacer, el tipo de actuación institucional, al respecto, es igualmente importante» (p.29).

Ahora bien, si se revisa esta situación a la luz de la deserción universitaria, Donoso y Schiefelbein (2007) consideran como variables de ello los factores atribuidos a:

- Características previas del sujeto y su éxito en los primeros años de universidad.

- Identificación de los aspectos a los cuales los estudiantes atribuyen importancia antes de graduarse. 
- Descripción de experiencias diseñadas para incrementar la retención.

- Análisis de resultados de procesos de innovación pedagógica y su impacto en la retención (como dimensión en pleno desarrollo).

En estos factores es importante considerar su rendimiento escolar previo, pues la literatura sostiene que la motivación hacia el logro y hacia el aprendizaje son factores clave (aunque no exclusivos) para un buen desempeño académico. Es por ello que a continuación se profundizará en este concepto.

\subsection{Motivación y éxito académico}

La motivación es el motor que mueve toda conducta, razón por la cual se provocan y generan cambios tanto a nivel académico como en la vida en general. No obstante, desde la perspectiva de algunos autores, «(...) el marco teórico-explicativo de cómo se produce la motivación, qué variables la determinan, cómo se puede mejorar desde la práctica docente (...) son cuestiones que dependen de la conceptualización teórica que se adopte» (Maquilón y Fuensanta 2011: 86).

A pesar de las discrepancias existentes en las teorías de la motivación, según lo expuesto por Maquilón y Fuensanta (2011), la mayoría de los especialistas coinciden en la definición de motivación como el «conjunto de procesos implicados en la activación, dirección y persistencia de la conducta» (Beltrán, 1993a; McClelland, 1989; y otros citados en Maquilón y Fuensanta, 2011: 87).

Respecto de la motivación por el rendimiento, Junco (2010) plantea que esta puede comprenderse como el intento de aumentar o mantener, lo más alto posible, la propia habilidad en todas aquellas actividades en las cuales se considera obligada una norma de excelencia y cuya realización, por tanto, puede lograrse o fracasar.

En este sentido, para Martínez (2011b) existe una directa relación entre la motivación y el rendimiento académico. Desde su perspectiva, numerosos estudios han incluido cierto número de variables explicativas que se han centrado en determinar cómo las variables personales (del profesor y del alumno), contextuales 
(especialmente vinculadas al ámbito familiar o del centro académico) y de la tarea (grado de dificultad, etc.) influyen en el rendimiento académico.

Para Martínez el rendimiento es un concepto complejo, multidimensional y polémico que depende de múltiples factores personales, contextuales y de la tarea, y la mayor parte de las investigaciones llevadas a cabo se han caracterizado por el uso -por no decir el abuso- de las calificaciones medias del estudiante como única medida del logro académico.

Por lo tanto, si bien es cierto es relevante considerar las calificaciones, no es posible sintetizar el logro académico solo a partir de estas. En consecuencia, el autor asume en su propuesta que «la motivación constituye un proceso de regulación, una competencia que incluye aspectos cognitivos, afectivos, sociales y conductuales» (Martínez 2011b:2). Por lo tanto, las calificaciones pueden ser consideradas como una referencia, pero un buen estudiante debe presentar características de automotivación. En otras palabras, el estudiante universitario debe ser autónomo, responsable y hacerse cargo de sí mismo, es decir, la motivación es propiamente suya. El autor confirma en sus investigaciones las siguientes características de los estudiantes que se automotivan:

- Poseen metacognición: planifican, organizan, controlan, evalúan $y$, en general, dirigen sus procesos mentales, sus emociones, sus conductas, hábitos y la propia observación.

- Usan estrategias cognitivas vinculadas con su trabajo.

- Poseen ciertas creencias motivacionales (intrínsecas y extrínsecas) y emociones adaptativas respecto de sí mismos y de la tarea (entusiasmo, gozo, satisfacción, etc.).

- Tienen sentido de autoeficacia.

- Planifican y controlan el tiempo y el esfuerzo, siendo persistentes y gestionando la ansiedad ante los exámenes.

- Usan estrategias profundas y significativas en lo que hacen: no son superficiales.

- Saben buscar y pedir ayuda. 
Por su parte, Manassero y Vázquez (1997; 1998) diseñaron una escala que mide la motivación, basada en la teoría de Weiner (atribución-emoción-acción). Esta escala ha inspirado diversos trabajos relacionados con la motivación y el rendimiento escolar, sin embargo, su aplicación y análisis en educación superior son recientes.

Un ejemplo para este nivel corresponde a la aplicación realizada por Bravo, González y Maytorena (2008), quienes concluyeron que los estudiantes de las carreras de Trabajo Social y Arquitectura atribuyen sus resultados académicos en situación de éxito a la «motivación e interés por la tarea/capacidad y esfuerzo»; los de Ingeniería en Sistemas de Información y Economía realizan sus atribuciones a partir de su «motivación de examen»; mientras que los de Arquitectura y Geología refieren mayormente la «motivación por el profesor». En el caso de situación de fracaso, los estudiantes de las carreras de Trabajo Social y Arquitectura realizan sus atribuciones a la «motivación de interés», «motivación por la tarea/capacidad», «de esfuerzo»y «de examen», mientras que las carreras de Enfermería y Economía se enfocan en la «motivación por parte del profesor».

Morales y Gómez (2009) reportan los resultados del estudio de validez de constructo y fiabilidad de una Escala Atribucional de Motivación de Logro (EAML), adaptada de Manassero y Vázquez (1997; 1998) para su aplicación en contextos educativos universitarios, en los cuales se implementan estrategias de aprendizaje colaborativo. Las autoras reemplazaron la dimensión original de «motivación de competencia del profesor» por la dimensión «motivación de interacción», en la cual se incorporaron aspectos relacionados con la percepción del estudiante acerca de la influencia de la interacción con sus pares y el profesor, en un contexto de aprendizaje colaborativo.

Las conclusiones de las autoras indican que cuando un alumno se siente responsable directo de sus logros académicos, mostrará mayor persistencia en la tarea, dado que relaciona esos logros con su propio interés y esfuerzo.

Sin duda, si algo en lo cual la literatura especializada en el tema concuerda es que el rendimiento, el aprendizaje y la motivación son 
tres importantes conceptos básicos en pedagogía y psicología. Ellos están estrechamente relacionados entre sí y contribuyen a explicar el ámbito de actividad de profesores y estudiantes (Junco, 2008). Al respecto se sostiene, incluso, que las posibilidades de aprendizaje y buen rendimiento son prácticamente imposibles sin una alta motivación.

De ahí la relevancia de profundizar en estas cuestiones en el contexto de los estudiantes universitarios chilenos, dado que los resultados obtenidos en la PSU se centran en la medición de los contenidos de enseñanza media, situación que podría invisibilizar otros aspectos relevantes a la hora de diseñar y poner en juego estrategias de selección que tengan como meta la retención y el egreso oportuno de las universidades.

\section{Metodología del estudio}

\subsection{Fase cualitativa}

Esta fase tiene como objetivo explicitar, desde el discurso de los estudiantes que son parte del programa propedéutico de la UCSH, el valor que otorgan a esta experiencia. Además, se busca hacer visibles las características personales que se encuentran como base de su alto rendimiento académico.

Como técnica de recolección de información se ha utilizado la entrevista en profundidad, ya que esta tiene como propósito central encontrar lo que es significativo e importante en la mente de los informantes. Es decir, sus perspectivas, interpretaciones, el modo en que ellos ven, clasifican y experimentan su propio mundo (Ruiz, 2003).

Con posterioridad a la especificación de las características de los actores, se construyó un protocolo de entrevista en el cual se establecieron los objetivos específicos y las dimensiones por cubrir. A partir de ello, se elaboraron preguntas que se sometieron a juicio de expertos. Con todo ello y las sugerencias realizadas, se construyó el protocolo definitivo de aplicación. 
Participaron en este proceso seis estudiantes que se encontraban cursando el programa propedéutico de la UCSH, por lo tanto se trata de jóvenes que cursaban $4^{\circ}$ año medio y se encontraban en el $7 \%$ superior del ranking en sus establecimientos, pertenecientes a los quintiles primero y segundo; todos ellos estaban matriculados en escuelas municipales.

Como se señaló inicialmente para el estudio de los discursos se utilizó el «análisis estructural», siguiendo las orientaciones de Martinic (1995; 2006). Según el autor, este tipo de análisis se basa en un procedimiento inspirado en la lingüística estructural, la que permite identificar, en un primer momento, las unidades básicas de sentido y las relaciones existentes entre ellas $y$, en un segundo momento, el método que organiza el movimiento de tales relaciones en un modelo de acción social (modelo actancial) que orienta la práctica de los sujetos enunciadores del discurso.

Los resultados recomponen las estructuras, identificando las realidades o temas que se asocian entre sí a través de relaciones de oposición o equivalencia. Luego se dinamizan las estructuras, distribuyendo las oposiciones y asociaciones identificadas en un modelo de acción social. Finalmente, se analizan las funciones simbólicas que asumen las distintas realidades o elementos considerados en el paso anterior, asumiendo que dicha organización y estructura da sentido a las prácticas o acciones que realizan los sujetos.

\subsection{Fase cuantitativa}

Esta fase tiene como propósito describir el perfil motivacional que presentan los estudiantes que ingresan a la universidad mediante el propedéutico versus los estudiantes que ingresan por la vía tradicional. Subyace la hipótesis en la cual se infiere que no existen diferencias entre ambos grupos y que estas diferencias solo estarían en el tipo de ingreso, ya que detrás del rendimiento escolar prevalece una alta motivación hacia el aprendizaje.

Se contempla el análisis de la motivación a través de dos escalas, siendo la primera la escala de Automotivación, AM4, diseñada por 
Martínez (2011a). En ella, el autor incorpora los factores denominados estrategias, regulación, planificación, refuerzos, cuidados y centrarse. Esta escala ha sido validada en el contexto del Espacio Europeo de Educación Superior y su propósito es estimar la relación entre sus factores y el rendimiento académico. Esta escala mide los procesos cognitivos, afectivos, relacionales y conductuales en estudiantes universitarios.

Por su parte, Manassero y Vázquez $(1997 ;$ 1998) diseñaron una escala que mide la motivación, basada en la teoría de Weiner (atribución-emoción-acción). En este modelo se analizan las atribuciones causales que guían y motivan la conducta. Los autores consideraron en su trabajo cinco factores explicativos como causas que se atribuyen al rendimiento: esfuerzo, tarea y capacidad, interés, exámenes y la percepción del profesor.

Ambas escalas fueron aplicadas a estudiantes pertenecientes a primer año en la UCSH. Se consideró a estudiantes tanto de ingreso tradicional (vía PSU), así como a estudiantes pertenecientes al programa de Bachillerato en Ciencias y Humanidades, cuyo ingreso es por medio del programa propedéutico.

La muestra quedó conformada por 198 estudiantes, quienes se encuentran en primer año en la UCSH. De este universo, 59 estudiantes (30\%) ingresaron a la universidad vía programa propedéutico y los 139 restantes (70\%) a través del ingreso regular vía PSU. Se presentaron 60 estudiantes (30\%) de sexo masculino y 138 de sexo femenino (69\%).

\section{Resultados}

\subsection{Fase cualitativa}

La estructura paralela (Figura 1) demuestra la organización de la representación que los estudiantes entrevistados tienen acerca de los sistemas de selección para el ingreso a la universidad. Los códigos de calificación atribuyen propiedades de un modo sistemático paralelo e inverso a los términos o realidades que forma el código de base. 
- Los códigos se construyen a través de dos principios que operan en forma simultánea. En primer lugar el principio de oposición o de disyunción y, en segundo lugar, el principio de unidad o de conjunción.

- Este principio de oposición será representado por una barra (/). Los términos y su denominador común se deducen a partir del material.

- El principio de conjunción será denominado totalidad y con él se da cuenta del eje semántico (T).

- En la disyunción construida por el locutor uno de los dos términos adquirirá el valor positivo (+) y el otro, por el mismo principio de oposición, tendrá el valor negativo (-).

\begin{tabular}{|l|l|}
\hline A, B & términos o realidades constituidas \\
\hline$/$ & relación de oposición o de disyunción \\
\hline T & Totalidad \\
\hline$-/+$ & Índice de valorización \\
\hline
\end{tabular}

En esta fase se realiza la construcción de estructuras describiendo las diferentes relaciones que se establecen entre los códigos construidos, es decir, se organiza el texto subyacente de un modo particular. Estas relaciones dan cuenta de la organización o estructura de las representaciones que son objeto de estudio y se organizan de la siguiente forma:

\begin{tabular}{|l|l|l|}
\hline c.b & Código de base & $\begin{array}{l}\text { Realidades o elementos discursivos materiales sobre los cuales los } \\
\text { hablantes construyen su discurso. }\end{array}$ \\
\hline c.c & $\begin{array}{l}\text { código de } \\
\text { calificación }\end{array}$ & $\begin{array}{l}\text { Apreciaciones o valoraciones que califican o atribuyen propiedades, } \\
\text { implícita o explícitamente, a términos o realidades que aparecen como } \\
\text { significantes en un texto. }\end{array}$ \\
\hline $\mathbb{I}$ & Implicancia & $\begin{array}{l}\text { Ambos conceptos establecen las relaciones lógicas y necesarias entre las } \\
\text { realidades que califican y aquellas que son calificadas. }\end{array}$ \\
\hline
\end{tabular}

En este caso, la totalidad de la calificación (TC) corresponde a los sistemas de selección para ingresar a la universidad. Los códigos de base corresponden a la PSU con valoración negativa y al programa propedéutico, con una valoración positiva.

En los discursos analizados la PSU demanda altos puntajes, lo que dificulta las posibilidades de ingreso a la universidad, ya que el 
éxito solo se basa en estos resultados. En consecuencia, esta modalidad de selección se asocia y valora negativamente como un sistema que segrega a los estudiantes que pertenecen a contextos vulnerables. Esto implica una limitación del sistema educativo en su conjunto.

Por su parte, el programa propedéutico y bachillerato es definido como un sistema que exige bajos puntajes y prioriza la selección a través de las notas obtenidas en la enseñanza media. Esta situación es valorada positivamente como una buena oportunidad para ingresar a la universidad, ya que disminuye las barreras derivadas de las exigencias de la PSU debido a que las calificaciones obtenidas son atribuidas a las características del estudiante; por lo tanto, con estas acciones la universidad es representada como un sistema que no excluye a las personas que provienen de colegios vulnerables y que, por diversas razones, no han tenido la preparación adecuada para la prueba. Para los entrevistados, los programas propedéuticos son un reflejo de un sistema educativo más democrático, en consecuencia, todas las universidades deberían contar con un sistema de selección como este.

En síntesis, se aprecia que los sistemas universitarios y sus políticas de acceso son representados como sistemas complejos, con altos niveles de segregación, en los cuales además existe una gran inequidad y escasas oportunidades para aquellos que presentan carencia de recursos económicos. En cambio, el programa propedéutico es valorado como un sistema que favorece el acceso a la universidad para aquellos estudiantes que provienen de contextos vulnerados y que, sin esta alternativa, tendrían como única opción entrar al mundo laboral. 
Figura 1: Estructura paralela

VÍAS DE INGRESO A LA EDUCACIÓN SUPERIOR

\begin{tabular}{|c|c|c|c|c|}
\hline & $(+)$ & & $(-)$ & $\mathrm{T}$ \\
\hline Cc6 & $\begin{array}{c}\mathrm{Al} \\
\text { Avance y democracia }\end{array}$ & 1 & $\begin{array}{c}\text { B1 } \\
\text { Limitación }\end{array}$ & $\begin{array}{c}\mathrm{TCl} \\
\text { Impacto }\end{array}$ \\
\hline \multicolumn{5}{|l|}{$\Uparrow$} \\
\hline Cc5 & $\begin{array}{c}\mathrm{A} 2 \\
\text { "No hay segregación con los otros } \\
\text { alumnos y la universidad confía en } \\
\text { esos alumnos" }\end{array}$ & 1 & $\begin{array}{c}\text { B2 } \\
\text { (Segregador para los estudiantes } \\
\text { de contextos vulnerables) }\end{array}$ & $\begin{array}{c}\mathrm{TC2} \\
\text { Características del } \\
\text { sistema }\end{array}$ \\
\hline \multicolumn{5}{|c|}{$\Uparrow$} \\
\hline Cc4 & $\begin{array}{c}\text { A3 } \\
\text { "Creo que la oportunidad } \\
\text { de estudiar gratis y hay que } \\
\text { aprovecharla" }\end{array}$ & 1 & $\begin{array}{c}\text { B3 } \\
\text { La oportunidad de estudiar } \\
\text { gratis debería beneficiar a más } \\
\text { estudiantes }\end{array}$ & $\begin{array}{c}\text { TC3 } \\
\text { Beneficios económicos }\end{array}$ \\
\hline \multicolumn{5}{|l|}{$\Uparrow$} \\
\hline $\mathrm{Cc} 3$ & $\begin{array}{c}\text { A 4 } \\
\text { Pocas, porque el éxito depende } \\
\text { del estudiante }\end{array}$ & 1 & $\begin{array}{c}\text { B4 } \\
\text { Muchas, porque el éxito } \\
\text { depende de los resultados PSU }\end{array}$ & $\begin{array}{c}\text { TC4 } \\
\text { Dificultades }\end{array}$ \\
\hline \multicolumn{5}{|l|}{$\Uparrow$} \\
\hline Cc2 & \begin{tabular}{|c|} 
A5 \\
$\begin{array}{c}\text { Buena oportunidad de selección } \\
\text { para acudir a la universidad } \\
\text { (notas). }\end{array}$ \\
\end{tabular} & 1 & $\begin{array}{c}\text { A5 } \\
\text { Dificultades para ingresar }\end{array}$ & $\begin{array}{c}\text { TC } 5 \\
\text { Oportunidades } \\
\text { de ingreso a la } \\
\text { universidad } \\
\end{array}$ \\
\hline \multicolumn{5}{|l|}{$\Uparrow$} \\
\hline $\mathrm{CCl}$ & $\begin{array}{l}\text { A6 } \\
\text { Baja }\end{array}$ & 1 & $\begin{array}{c}\text { B6 } \\
\text { Alta }\end{array}$ & $\begin{array}{c}\text { TC6 } \\
\text { Puntajes }\end{array}$ \\
\hline \multicolumn{5}{|l|}{$\Uparrow$} \\
\hline $\mathrm{CB}$ & $\begin{array}{c}\text { A } \\
\text { Propedéutico }\end{array}$ & 1 & $\begin{array}{c}\text { B } \\
\text { PSU }\end{array}$ & $\begin{array}{c}\text { TC } \\
\text { Sistemas de selección } \\
\text { universitaria }\end{array}$ \\
\hline
\end{tabular}

$\mathrm{cAcB}=$ Realidades o términos de calificación

$\mathrm{A}, \mathrm{B}=$ Términos o realidades $\mathrm{u}$ objetos significantes

$\mathrm{cc}=$ Códigos de calificación o implicación

$\mathrm{cb}=$ Código de base

$\mathrm{Tc}=$ Totalidad calificativa

$\mathrm{Tb}=$ Totalidad de base

$\Uparrow=$ Implicación lógica

La siguiente estructura (Figura 2) cruza los ejes de actitud hacia los estudios y las calificaciones que se obtienen. Los jóvenes entrevistados declaran que este rendimiento se debe a su esfuerzo y autoexigencia (campo semántico n II). Marcan la diferencia de sus acciones y actitudes respecto de las que realizan otros compañeros que no se toman con seriedad los estudios y que, más bien, son una barrera 
para el aprendizaje de aquellos que sí desean progresar y aprender. El grupo al que aluden obtiene malas calificaciones producto de su relajo e indiferencia (campo semántico ${ }^{\circ}$ III). No obstante, también en sus discursos se hace mención a aquellos pares que aun cuando se esfuerzan y se autoexigen, no logran obtener buenos resultados (campo semántico $\mathrm{n}^{\circ} \mathrm{I}$ ). Este caso da cuenta de aquellos jóvenes que serían los menos beneficiados, ya que sus oportunidades son incluso más limitadas. En el campo $n^{\circ}$ IV se encuentran aquellos estudiantes que se relajan, son indiferentes y obtienen buenas calificaciones. Es importante destacar que esta calificación no se encontró en los discursos que hasta el momento se han analizado. Sin embargo, la presente investigación se encuentra en proceso y aún pueden aparecer estas perspectivas discursivas.

Figura 2: Estructura cruzada

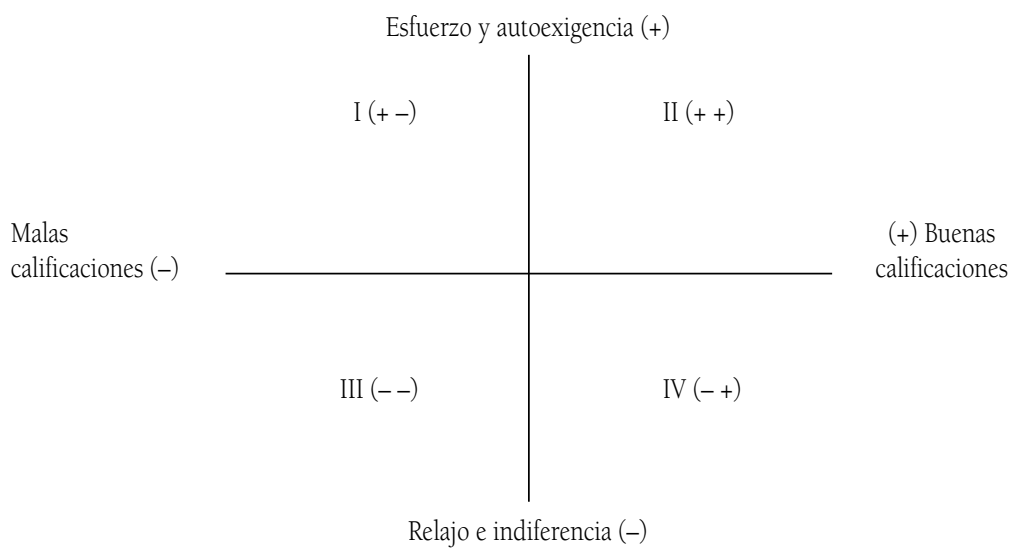

\subsection{Fase cuantitativa}

\subsubsection{Análisis descriptivo}

El cuestionario de automotivación tiene un puntaje mínimo de 25 puntos y un máximo de 120 puntos. En la aplicación realizada el puntaje mínimo corresponde a 15 puntos y el máximo a 75. La media es de 60 puntos $(M=60,32 ; S D=9,06)$. En la escala de Manassero y Vázquez (1997; 1998) el mínimo corresponde a 83 puntos, en tanto que el puntaje máximo alcanza a 189. La media es de 147 $(M=147,44 ; S D=19,242)$. 
Tabla 1: Estadísticos descriptivos

\begin{tabular}{|l|c|c|c|c|c|}
\hline Estadísticos descriptivos \\
\hline & $\mathrm{N}^{\circ}$ & Mínimo & Máximo & Media & Desv. típ. \\
\hline Puntaje total de automotivación & 198 & 15 & 75 & 60,32 & 9,069 \\
\hline Puntaje total de motivación de logro & 198 & 83 & 189 & 147,44 & 19,242 \\
\hline N válido (según lista) & 198 & & & & \\
\hline
\end{tabular}

\subsubsection{Estimación de la confiabilidad}

La confiabilidad medida a través de Alfa de Crombach reporta $\alpha$ : 0,98 para la escala de automotivación (Martínez, 2011a) y $\alpha: 0,85$ para la escala de motivación de logro (Manassero y Vázquez, 1996; 1997). En consecuencia, para ambas escalas se estima que exista una buena confiabilidad.

\subsubsection{Comparación de confiabilidad}

A continuación se presentan los resultados obtenidos en las aplicaciones originales realizadas por sus respectivos autores y los resultados obtenidos en la investigación correspondiente a la UCSH (Román, 2012).

El perfil dimensional evidencia que en los resultados de la escala AM4 la confiabilidad por subescala es similar a la obtenida por Martínez (2011a) en su aplicación original. Estos valores son los siguientes:

Tabla 2. Análisis comparativo Alfa de Crombach AM4

\begin{tabular}{|l|c|c|}
\hline \multicolumn{3}{|c|}{ Análisis comparativo Alfa de Crombach AM4 } \\
\hline & Martínez & Román \\
\hline Estrategias & 0,80 & 0,79 \\
\hline Regulación & 0,81 & 0,85 \\
\hline Planificación & 0,79 & 0,84 \\
\hline Refuerzos & 0,73 & 0,62 \\
\hline Cuidados & 0,72 & 0,59 \\
\hline Centrarse & 0,73 & 0,78 \\
\hline
\end{tabular}

Los resultados obtenidos en la aplicación de la escala de motivación de logro, EML, evidencian que la confiabilidad para la subescala denominada "examen» es baja; por lo tanto, es recomendable evitar realizar conclusiones para este nivel. Además, la 
subescala "profesor» presenta una confiabilidad baja en comparación con los resultados obtenidos en las otras subescalas, sin embargo, es posible considerar su valor como aceptable.

Tabla 3: Análisis comparativo Alfa de Crombach escala EML

\begin{tabular}{|l|c|c|}
\hline \multicolumn{3}{|c|}{ Análisis comparativo Alfa de Crombach escala EML } \\
\hline & Manassero y Vázquez & Román \\
\hline Interés & 0,79 & 0,72 \\
\hline Tarea & 0,78 & 0,62 \\
\hline Esfuerzo & 0,80 & 0,79 \\
\hline Examen & 0,64 & 0,18 \\
\hline Profesor & 0,58 & 0,49 \\
\hline
\end{tabular}

4.2.4. Comparación de medias entre estudiantes de programa propedéutico versus estudiantes de ingreso tradicional A continuación se comparan las medias obtenidas por subescalas en la escala AM4 entre los estudiantes que ingresan a través del programa propedéutico versus los estudiantes que ingresan por la vía tradicional. Los resultados evidencian que las medias obtenidas entre ambos grupos son similares.

En el grupo de estudiantes de programa propedéutico la media más alta corresponde a la escala «planificación» $(20,47)$, mientras que la media más baja corresponde a «centrarse» $(8,27)$. Estas medias son similares a las obtenidas por los estudiantes que ingresan por la vía tradicional. Sin embargo, en este grupo los valores son levemente superiores.

Tabla 4: Análisis comparativo de medias AM4

\begin{tabular}{|l|c|c|}
\hline \multicolumn{3}{|c|}{ Análisis comparativo de medias AM4 } \\
\hline & Propedéutico & Regular \\
\hline Estrategias & 19,68 & 20,02 \\
\hline Regulación & 20,14 & 19,87 \\
\hline Planificación & 20,47 & 21,00 \\
\hline Refuerzos & 15,39 & 15,05 \\
\hline Cuidados & 12,05 & 12,12 \\
\hline Centrarse & 8,27 & 8,16 \\
\hline
\end{tabular}

En el caso de los resultados de la escala de motivación de logro, EML, se aprecia que tal como sucedió con la escala de automotivación, 
las medias entre estudiantes que ingresan a la universidad vía propedéutico versus aquellos que ingresan vía tradicional son similares. En este caso, la media más alta corresponde a la subescala de «interés» (M 38,51), mientras que la media más baja corresponde a la subescala de «examen». No obstante, como se describió en el análisis de confiabilidad, no es posible hacer conclusiones en este nivel, en consecuencia, se estima que la media más baja en este grupo de estudiantes chilenos corresponde a la subescala «profesor».

Tabla 5: Análisis comparativo media escala EML

\begin{tabular}{|l|c|c|}
\hline \multicolumn{3}{|c|}{ Análisis comparativo Media escala EML } \\
\hline & Propedéutico & Regular \\
\hline Interés & 38,51 & 39,02 \\
\hline Tarea & 31,92 & 31,27 \\
\hline Esfuerzo & 29,24 & 29,07 \\
\hline Examen & 11,83 & 10,67 \\
\hline Profesor & 13,64 & 13,47 \\
\hline
\end{tabular}

\section{Conclusiones}

Tanto la escala AM4 como la EML son confiables para medir la motivación en el contexto de la educación superior chilena, sin embargo, la segunda subescala correspondiente a «examen» arroja una confiabilidad baja, razón por la cual es recomendable eliminarla, o bien, reemplazarla para cumplir con el objetivo del instrumento (ver propuesta de Morales y Gómez, 2009).

Considerando los resultados obtenidos en la aplicación de la escala AM4 (Martínez, 201la) se evidencia que los estudiantes que participaron en la investigación presentan un alto grado de planificación, es decir, fijan objetivos y prioridades, buscan sentido en lo que hacen y se centran en terminar lo que han comenzado. Por otra parte, el resultado más descendido se encuentra en el nivel denominado «centrarse». Esta subescala constituye una medida metacognitiva de concentración y autoprogramación para realizar planes y cumplirlos con una actitud positiva.

Respecto de los resultados obtenidos en la aplicación de la escala EML (Manassero y Vázquez, 1997; 1998), se aprecia que los estudiantes 
participantes en la investigación presentan el mayor puntaje a nivel de interés, en consecuencia, sus calificaciones dependen principalmente de esta variable. Por su parte, el puntaje menor estuvo en la subescala «profesor», lo cual implica que las atribuciones de los estudiantes a la suerte y sesgos del profesor, son menores.

Es importante considerar que los resultados antes expuestos abarcan el total de la muestra, ya que los jóvenes que ingresan a la UCSH vía resultados PSU, o bien, vía programa propedéutico obtuvieron prácticamente los mismos valores a nivel de escalas y subescalas. En consecuencia, se confirma la hipótesis de entrada en la cual se sostiene que ambos grupos presentan perfiles motivacionales similares y que la diferencia solo radica en el tipo de ingreso, es decir, tradicional o vía propedéutico.

Mirado de esta forma, se sostiene que detrás del buen rendimiento académico en los estudiantes que ingresan a la universidad por la vía del programa propedéutico existen habilidades no cognitivas referidas a la motivación hacia el logro, las que guían y orientan su conducta (Gallegos y Meneses 2007; Contreras, Gallegos y Meneses 2009; Meneses y Toro 2012).

Si se considera que la retención y egreso oportuno no solo dependen de los resultados obtenidos en la PSU, se aprecia que los jóvenes que ingresan a la universidad vía programa propedéutico presentan un perfil que da cuenta de una motivación intrínsecamente alta, ya que el rendimiento se asocia de forma particular a sus propias acciones. No obstante, también es importante seguir avanzando en estas cuestiones, ya que se considera una limitante de este estudio el puntaje promedio obtenido en la PSU por los estudiantes que ingresan a la UCSH (528) versus los estudiantes del bachillerato (502). Entonces, es recomendable realizar nuevas investigaciones con estudiantes que obtengan altos puntajes en esta prueba, a fin de seguir sustentando esta hipótesis con muestras que presenten mayor heterogeneidad.

En términos cualitativos, a partir del análisis estructural de discurso realizado (Martinic, 1995; 2006) se concluye que los 
estudiantes que ingresan a través del programa propedéutico realizan una valoración positiva de esta opción de acceso, ya que describen el programa como una acción que hace más justo y equitativo el sistema educativo: si solo se consideraran los resultados obtenidos en la PSU, sus posibilidades de cursar estudios universitarios se habrían visto limitadas.

Cuando se trata de especificar las características que están detrás de sus altas calificaciones, los jóvenes expresan que son personas esforzadas, con metas claras, que se autoexigen a sí mismas. Es decir, existen rasgos de perfeccionismo en sus tareas y las acciones que deciden emprender. Sin embargo, cuando valoran los programas propedéuticos el foco del discurso se encuentra en la oportunidad para «ellos», es decir, los estudiantes en desventaja social, pero queda fuera su propio mérito y las características que los han llevado a ser los mejores alumnos de sus respectivos establecimientos.

En este sentido se infiere que existe la posibilidad de que, aun cuando los jóvenes ingresen a la universidad, no se sientan merecedores de este ingreso, ya que el valor central de los programas propedéuticos se atribuye a las universidades que les abren una posibilidad de acceso. Queda excluido, al menos en esta parte de la investigación, el valor propio como estudiante con talento que se ha destacado por su rendimiento académico.

Por todo lo anteriormente expuesto, se concluye que los programas propedéuticos constituyen una acción que favorece el ingreso a la educación superior y que todas las universidades deberían tener vías de acceso iguales o similares, ya que el talento académico de los estudiantes, expresado a través del ranking de notas utilizado para su selección en estos programas, da cuenta de habilidades necesarias para cursar estudios superiores. Se trata, pues, de características personales que tienen como sustrato la disciplina, metas claras, planificación y una alta motivación.

Sin embargo, también es importante que las instituciones de educación superior y los docentes no descansen exclusivamente en los méritos de los estudiantes, ya que si estos no cuentan con el apoyo 

LA UNIVERSIDAD A TRAVÉS DEL PROGRAMA PROPEDÉUTICO: UN ANÁLISIS DESDE LA MOTIVACIÓN Y EL DISCURSO DE LA UCSH - C. Román

necesario pueden abandonar sus carreras, o bien, presentar tiempos de graduación superior al de sus pares que ingresan vía PSU (Gil y Del Canto, 2012). Esta situación es compleja, ya que la mayoría de los jóvenes financia sus estudios con becas y créditos estatales que no cubren los años de atraso. Por lo tanto, urge seguir indagando respecto de los apoyos que se les entrega una vez que egresan de los programas de bachillerato e ingresan a las carreras profesionales.

También es importante considerar que en el contexto chileno la inclusión en este nivel se encuentra limitada si no se instalan políticas de acceso que permitan que todos los sectores sociales, capacidades y talentos se encuentren representados (Chiroleu, 2009), pues tal como se encuentra la situación hoy, el sistema selecciona por resultados académicos (PSU, NEM, ranking) y, por supuesto, en un sector con capacidad para pagar (Rama, 2006).

Además, tal como se obtuvo del discurso de los participantes en esta parte de la investigación, también existen otros jóvenes que se esfuerzan y autoexigen y aun así no logran obtener altas calificaciones. En este caso vale la pena hacerse las siguientes reflexiones finales: ¿qué pasa con ellos?, ¿la universidad debe estar concebida solo para los estudiantes que obtienen buenos resultados en la PSU o que tienen buen rendimiento?, ¿cómo se puede favorecer el acceso, retención y egreso oportuno de diversas capacidades, diversos talentos y diversas clases sociales?

\section{Referencias}

Abarca, V.; Assaél, J.; Brzovic, D.; Caldichoury, J.; Cornejo, R.; González, J.; Redondo, J.; Sánchez, R. y M. Sobarzo, (2009) De actores secundarios a protagonistas. Disponible en: http://www.opech.cl/Libros/doc5.pdf

Acuña, F.; Arévalo, C.; Baeza, F.; Fredes, D.; González, D.; Herrada, J.; Mendoza, M.; Osorio, S.; Quiroga, L.; Sánchez, R.; Sobarzo, M. y N. Zamorano, (2009) Acceso a la educación superior: el mérito y la (re) producción de la desigualdad. Grupo de investigación CESCC OPECH. Disponible en http://www.opech.cl/inv/analisis/acceso.pdf

Bourdieu, P. y J. Passeron, (1964) Los herederos: los estudiantes y la cultura. Buenos Aires: Siglo Veintiuno. 
Bravo, A., González, D. y M. Maytorena, (2008) Motivación de logro en situaciones de éxito y fracaso académico de estudiantes universitarios. Disponible en:http://www.comie.org.mx/congreso/memoriaelectronica/v10/pdf/ area_tematica_01/ponencias/0762-F.pdf

Castillo, J. y G. Cabezas, (2010) Caracterización de jóvenes de primera generación en educación superior. Nuevas trayectorias hacia la equidad educativa. Calidad en la educación (32), pp. 54-74.

Chiroleu, A., (2009) La inclusión en educación superior como política pública: tres experiencias en América Latina. Revista iberoamericana de educación, 48 (5). Disponible en: http://www.rieoei.org/ deloslectores/2740Chiroleu.pdf

Contreras, D.; Gallegos, S. y F. Meneses, (2009) Determinantes de desempeño universitario: ¿importa la habilidad relativa?, Calidad en la educación (30), pp. 18-48.

Departamento de Evaluación, Medición y Registro Educacional DEMRE. (2011).Disponible en: http://www.demre.cl/demre.htm

Donoso, S. y E. Schiefelbein, (2007) Análisis de los modelos explicativos de retención de estudiantes en la universidad: una visión desde la desigualdad social. Estudios pedagógicos de Valdivia, 33 (1), pp. 7, 27.

Gallegos, S. y F. Meneses, (2007) ¿Es eficiente el sistema de ingreso a la universidad? El uso del ranking en la Universidad Católica de Chile. Disponible en: http://www.cai.cl/web/web2/images/documentos/ Docuementos_Contingencia/AFI/afi.doc2.pdf

Gil, F. y J. Bachs, (2008) Una experiencia exitosa por una educación más inclusiva. Disponible en: http://www.propedeutico.cl//centroDocumentacion.aspx

Gil, F. y C. Del Canto, (2012) El caso del programa propedéutico en la Universidad de Santiago. Pensamiento educativo, 49 (2), pp. 65-83.

Junco, I., (2010) La motivación en el proceso de enseñanza-aprendizaje. Temas para la educación, 9. Disponible en: http://www2.fe.ccoo.es/andalucia/ docu/p5sd7327.pdf

Kremerman, M., (2007) El desalojo de la universidad pública. Disponible en: http://www.opech.cl/bibliografico/doc_movest/el_desalojo_ universidad.pdf

Killonen, P., (2012) La importancia de la educación superior y el rol de los atributos no cognitivos en el éxito en dichas instituciones. Pensamiento educativo, 49 (2), pp. 84-100.

Leyton, D., Fuenzalida, Vásquez, A y V. Fuenzalida, (2012) La experiencia de estudiantes de contextos vulnerables en diferentes instituciones de 
educación superior (IESU): resultados de una investigación. Calidad de la educación (37), pp 62-97. Disponible en: http://www.cned.cl/ public/Secciones/SeccionRevistaCalidad/doc/74/cse_articulo1044.pdf

Manassero, M.A. y A. Vázquez, (1998) Validación de una escala de motivación de logro. Psicothema, 10, (2), pp. 333-351.

Manassero, M.A. y A. Vázquez, (1997) Análisis empírico de dos escalas de motivación escolar. Revista electrónica de motivación y emoción, 3 (56). Disponible en: http://reme.uji.es/articulos/amanam5171812100/ texto.html

Manzi, J., (2012 ¿Equidad en las pruebas o en la admisión? Disponible en: http://www.propedeutico.cl/publicacion.aspx?id=160

Maquilón, J. y P. Fuensata, (2011) Influencia de la motivación en el rendimiento académico de los estudiantes de formación profesional. REIFOP, 14, (1). Disponible en: http://www.aufop.com/aufop/ uploaded_files/articulos/1301588086.pdf

Martínez, J., (2011a) Diseño y validación del cuestionario de automotivación am-24 en el marco del Espacio Europeo de Educación Superior. Cuadernos de educación y desarrollo, España, 3, (27). Disponible en: http://www.eumed.net/rev/ced/27/jamg.htm

Martínez, J., (2011b) Automotivación y rendimiento académico en el Espacio Europeo de Educación Superior. Cuadernos de educación y desarrollo, 3, (28). Disponible en: http://www.eumed.net/rev/ced/28/jamg.htm

Martinic S., (1995) Principios culturales de la demanda social por educación. Un análisis estructural. Pensamiento educativo, 16, (1), pp. 313-39.

Martinic, S., (2006) El estudio de las representaciones y el análisis estructural del discurso. En Canales, M. (ed.). Metodologías de investigación social. Santiago, (pp. 299-320). Santiago, Chile: Lom.

Meneses, F. y J. Toro, (2012) Predicción de notas en Derecho de la Universidad de Chile: isirve el ranking? Revista_isees (10), pp. 43-58.

Morales, P. y V. Gómez, (2009) Adaptación de la escala atribucional de logro de Manassero y Vázquez. Investigación pedagógica, 12, (3), pp. 23-52.

Organización para la Cooperación y el Desarrollo Económico y Banco Mundial, (2009) Revisión de políticas nacionales de educación: la Educación Superior en Chile. Disponible en:http://www7.uc.cl/webpuc/piloto/ pdf/informe_OECD.pdf

Rama, C., (2006) La tercera reforma en educación superior en América Latina y el Caribe: masificación, regulaciones e internacionalización. Revista educación y pedagogía, 18,(46). 
Redondo, J., (2009) La educación chilena en una encrucijada histórica. Diversial, Disponible en: http://www.cidpa.cl/?p=308

Román, C., (2012) ¿Qué hay detrás del buen rendimiento escolar en los estudiantes que ingresan a la universidad a través del Programa Propedéutico de la UCSH (Chile)? Educere, (55), pp. 331-338. Disponible en: http://www.redalyc.org/pdf/356/35626140011.pdf

Ruiz, J., (2003) El diseño cualitativo. Disponible en: http://www. investigacioncualitativa.cl/2008/01/introduccin-la-investigacincualitativa.html

Scheele, J. y E. Treviño, (2012) Oportunidades de movilidad educacional y social en Chile: el propedéutico UNESCO. En Román C., Maureira O. y Catrileo C. (Eds.). Contextos formativos y sociales de programas propedéuticos en Chile: una aproximación a sus principales características y factores. Santiago, Chile: Ediciones Universidad Católica Silva Henríquez.

Sverdlick, I.; Ferrari P. y A. Jaimovich, (2005) Desigualdad e inclusión en la educación superior. Un estudio comparado en cinco países de América Latina. Disponible en:http://www.opech.cl/educsuperior/politica_ acceso/estudio_intersindical_latinoamericano_2005.pdf

Urra, J., (2012) La movilización estudiantil chilena en 2011: una cronología. OSAL, 13, (31). Disponible en: http://biblioteca.clacso.edu.ar/clacso/ osal/20120417105250/OSAL31.pdf

Recibido: 29/11/2012

Aceptado: 9/05/2013 